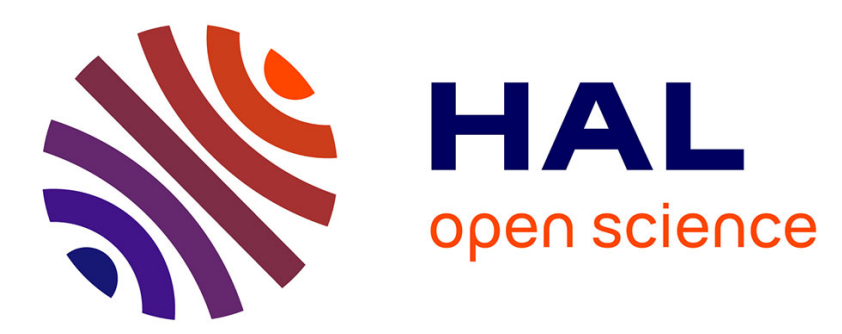

\title{
Ab initio based calculations of the thermal conductivity at the micron scale
}

Laurent Chaput, Jérôme Larroque, Philippe Dollfus, Jérôme Saint-Martin, David Lacroix

\section{- To cite this version:}

Laurent Chaput, Jérôme Larroque, Philippe Dollfus, Jérôme Saint-Martin, David Lacroix. Ab initio based calculations of the thermal conductivity at the micron scale. Applied Physics Letters, 2018, 112 (3), pp.033104. 10.1063/1.5010959 . hal-01689364

\section{HAL Id: hal-01689364 https://hal.science/hal-01689364}

Submitted on 1 Feb 2018

HAL is a multi-disciplinary open access archive for the deposit and dissemination of scientific research documents, whether they are published or not. The documents may come from teaching and research institutions in France or abroad, or from public or private research centers.
L'archive ouverte pluridisciplinaire HAL, est destinée au dépôt et à la diffusion de documents scientifiques de niveau recherche, publiés ou non, émanant des établissements d'enseignement et de recherche français ou étrangers, des laboratoires publics ou privés. 


\section{Ab initio based calculations of the thermal conductivity at the micron scale}

Laurent Chaput, Jérôme Larroque, Philippe Dollfus, Jérôme Saint-Martin, and David Lacroix

Citation: Appl. Phys. Lett. 112, 033104 (2018);

View online: https://doi.org/10.1063/1.5010959

View Table of Contents: http://aip.scitation.org/toc/apl/112/3

Published by the American Institute of Physics

\section{Articles you may be interested in}

Quantum ballistic transport in strained epitaxial germanium

Applied Physics Letters 111, 233512 (2017); 10.1063/1.5008969

Giant tunnel magnetoresistance in polycrystalline magnetic tunnel junctions with highly textured $\mathrm{MgAl}_{2} \mathrm{O}_{4}(001)$ based barriers

Applied Physics Letters 112, 022408 (2018); 10.1063/1.5013076

Tuning the interfacial charge, orbital, and spin polarization properties in $\mathrm{La}_{0.67} \mathrm{Sr}_{0.33} \mathrm{MnO}_{3} / \mathrm{La}_{1-x} \mathrm{Sr}_{x} \mathrm{MnO}_{3}$ bilayers

Applied Physics Letters 112, 032401 (2018); 10.1063/1.5011172

Photothermoelastic contrast in nanoscale infrared spectroscopy

Applied Physics Letters 112, 033105 (2018); 10.1063/1.4985584

Plasmonic micropillars for precision cell force measurement across a large field-of-view

Applied Physics Letters 112, 033701 (2018); 10.1063/1.5005525

Enhancement of average thermoelectric figure of merit by increasing the grain-size of $\mathrm{Mg}_{3.2} \mathrm{Sb}_{1.5} \mathrm{Bi}_{0.49} \mathrm{Te}_{0.01}$ Applied Physics Letters 112, 033903 (2018); 10.1063/1.5016488

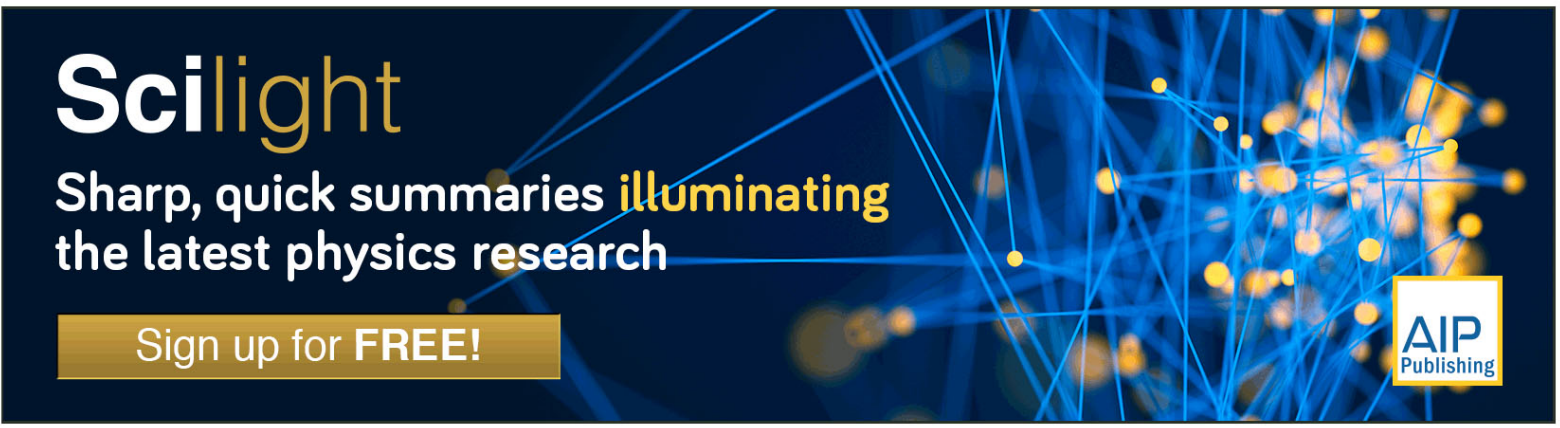




\title{
$A b$ initio based calculations of the thermal conductivity at the micron scale
}

\author{
Laurent Chaput, ${ }^{1, a)}$ Jérôme Larroque, ${ }^{2}$ Philippe Dollfus, ${ }^{2}$ Jérôme Saint-Martin, ${ }^{2}$ \\ and David Lacroix ${ }^{1}$ \\ ${ }^{1}$ Université de Lorraine, LEMTA, Centre National de la Recherche Scientifique, Unité Mixte de Recherche \\ 7563, BP 70239, 54506 Vandouvre Cedex, France \\ ${ }^{2}$ Center for Nanosciences and Nanotechnology, CNRS UMR 9001, Université Paris Saclay, Bât. 220, \\ F-91405 Orsay cedex, France
}

(Received 28 October 2017; accepted 2 January 2018; published online 17 January 2018)

\begin{abstract}
Heat transport in bulk semiconductors is well understood, and during the last few years, it has been shown that it can be computed accurately from ab initio calculations. However, describing heat transport in micro- and nanodevices used in applications remains challenging. In this paper, we propose a method, based on the propagation of wave packets, for solving the phonon Boltzmann transport equation parametrized with ab initio calculations. It allows computing the thermal conductivity of micro- and nano-sized systems, without adjustable parameters, and for any materials. The accuracy and applicability of the method are demonstrated by computing the cross plane thermal conductivity of cubic and hexagonal silicon thin films as a function of their thickness. Published by AIP Publishing. https://doi.org/10.1063/1.5010959
\end{abstract}

During the last few years, it has become possible to compute the lattice thermal conductivity of bulk semiconductors with good accuracy. ${ }^{1-3}$ Basically, information about the chemical binding is retrieved from density functional calculations, ${ }^{4-7}$ enabling building and solving the phonon Boltzmann transport equation. From the solution of that equation, the lattice thermal conductivity can then be computed without simplifying assumptions. The agreement of those calculations with experiments, for example, shown in Fig. 1(c) of Ref. 8, is remarkable.

The above mentioned calculations are performed for bulk crystals. However, many current applications require the study of heat transport in systems of finite dimensions, eventually of nanoscale size. It is true for spintronics, where heat transport needs to be described at the nano/micron scale to evidence the competing effects of thermal and magnetic interactions, ${ }^{9}$ or in thermoelectricity where heat transport is degraded by inserting nanoinclusions in a bulk material to increase its figure of merit. ${ }^{10}$

For finite sized systems, the current situation for the computation of thermal conductivity is much less satisfactory than for bulk crystals. This is mostly because the translational periodicity of the crystals is broken by the system boundaries, depriving us from easy solution to the Schrödinger and Boltzmann equations in reciprocal space. Two strategies are then possible to overcome this difficulty. On the one hand, it is possible to perform all calculations in direct space. This is the strategy used in molecular dynamics calculations. Unfortunately, the physical picture is blurred in this approach since collective modes are absent from the formalism. Another choice is to use a mixed representation between direct and reciprocal space. It is the strategy commonly used when the Boltzmann equation is parametrized using phonon wave properties, like frequency, group velocity, and lifetime, but solved in direct space to take care of the boundary conditions. This approach has been used several times in the past, with great success. ${ }^{11-14}$ However, it is often

a)Electronic mail: laurent.chaput@univ-lorraine.fr used with simplifying models, where, for example, an isotropic spectrum is assumed for the phonon dispersion, and an empirical lifetime is used. Phonon properties obtained from $a b$ initio calculations have been used only very recently. ${ }^{15}$

In this letter, we report a strategy to solve a Boltzmann equation parametrized with $a b$ initio calculations, in micronsize systems. It has been implemented in a computing code and applied to solve the Boltzmann equation, and therefore to compute the thermal conductivity, for any material and film thickness.

This letter is organized as follows. We first review the basic hypothesis of the method, and then we detail the computation strategy we propose. Finally, our computer code is used to compute heat transport across a thin film of cubic and hexagonal silicon. The results of those calculations are discussed, evidencing the peculiarities of each system.

When the system considered is out of thermodynamical equilibrium, the occupation function $n_{\mathbf{q} j}$ of the phonon modes $\mathbf{q} j$ is not known and must be determined. Within a semiclassical approximation, this can be done using the Boltzmann transport equation

$$
\frac{\partial n_{\mathbf{q} j}}{\partial t}(\mathbf{r}, t)+\mathbf{v}_{\mathbf{q} j} \cdot \frac{\partial n_{\mathbf{q} j}}{\partial \mathbf{r}}(\mathbf{r}, t)=\left.\frac{\partial n_{\mathbf{q} j}}{\partial t}\right|_{c o l}(\mathbf{r}, t),
$$

where $\mathbf{v}_{\mathbf{q} j}=\partial \omega_{\mathbf{q} j} / \partial \mathbf{q}$ is the phonon group velocity for wavevector $\mathbf{q}$ and polarization $j$ and $\partial n_{\mathbf{q} j} /\left.\partial t\right|_{c o l}$ is the rate of change in the occupation function due to collisions. Once the occupation function is obtained from this equation, the heat flux density, $\mathbf{J}=1 / V \sum_{\mathbf{q} j} \hbar \omega_{\mathbf{q} j} \mathbf{v}_{\mathbf{q} j} n_{\mathbf{q} j}$, where $V$ is the system volume, can be computed. This allows evaluating a thermal conductivity as $\kappa=|J| /|\nabla T|$, for a given temperature gradient $\nabla T$.

In general, the collision integral is decomposed as

$$
\left.\frac{\partial n_{\mathbf{q} j}}{\partial t}\right|_{c o l}(\mathbf{r}, t)=-\frac{n_{\mathbf{q} j}(\mathbf{r}, t)-n_{\mathbf{q} j}^{0}(\mathbf{r}, t)}{\tau_{\mathbf{q} j}}+C\left[n_{\mathbf{q} j}\right],
$$

where $n_{\mathbf{q} j}^{0}$ is the Bose Einstein occupation function, $\tau_{\mathbf{q} j}$ the lifetime of mode $\mathbf{q} j$, and $C\left[n_{\mathbf{q} j}\right]$ a correction to the exponential 
decays toward the equilibrium controlled by $\tau_{\mathbf{q} j}$. The explicit expression of the linear functional $C\left[n_{\mathrm{q} j}\right]$ is given by the collision operator $\Omega$ defined in Ref. 1 . It can be seen that it becomes zero if one assumes that the scattering events experienced by phonons do not change the occupation of modes $\mathbf{q}^{\prime} j^{\prime}$ for $\mathbf{q}^{\prime} j^{\prime} \neq \mathbf{q} j$. This is known as the single mode relaxation time approximation. This approximation will be used in the below paragraph, since it was shown to be accurate within $15 \%$ for silicon in Ref. 1. If this approximation allows saving computational time in applications, it is not an essential simplification in our approach. Therefore, the formalism will be derived for a general collision integral whenever possible.

Within the single mode relaxation time approximation, the Boltzmann equation is parametrized using the spectral parameters, $\omega_{\mathbf{q} j}, \mathbf{v}_{\mathbf{q} j}$, and $\tau_{\mathbf{q} j}$, which are material-dependent quantities. In the below paragraph, heat propagation in a silicon thin film will be considered. Therefore, $\omega_{\mathbf{q} j}, \mathbf{v}_{\mathbf{q} j}$, and $\tau_{\mathbf{q} j}$ have been computed using ab initio calculations for cubic silicon, and hexagonal silicon as well, to discuss the effects of material anisotropy. The details of the ab initio calculations are given in the supplementary material.

The characteristic lines ${ }^{16}$ of Eq. (1) are given by

$$
\frac{d \mathbf{r}(t)}{d t}=\mathbf{v}_{\mathbf{q} j} \Longleftrightarrow \mathbf{r}(t)=\mathbf{r}\left(t_{0}\right)+\mathbf{v}_{\mathbf{q} j}\left(t-t_{0}\right) .
$$

Along these lines, the total variation ${ }^{16}$ of phonon occupation function is given by

$$
\frac{d}{d t} n_{\mathbf{q} j}(\mathbf{r}(t), t)=\left.\frac{\partial n_{\mathbf{q} j}}{\partial t}\right|_{c o l}(\mathbf{r}(t), t)
$$

and therefore,

$$
n_{\mathbf{q} j}(\mathbf{r}(t), t)=n_{\mathbf{q} j}\left(\mathbf{r}\left(t_{0}\right), t_{0}\right)+\left.\int_{t_{0}}^{t} d s \frac{\partial n_{\mathbf{q} j}}{\partial t}\right|_{c o l}(\mathbf{r}(s), s) .
$$

Taking $t=t_{0}+\delta t$ with a time step $|\delta t| \ll t_{0}$

$$
\begin{aligned}
& n_{\mathbf{q} j}\left(\mathbf{r}\left(t_{0}\right)+\mathbf{v}_{\mathbf{q} j} \delta t, t_{0}+\delta t\right) \\
& \quad=n_{\mathbf{q} j}\left(\mathbf{r}\left(t_{0}\right), t_{0}\right)+\left[\left.\frac{\partial n_{\mathbf{q} j}}{\partial t}\right|_{c o l}\left(\mathbf{r}\left(t_{0}\right), t_{0}\right)\right] \delta t .
\end{aligned}
$$

This equation allows propagating the phonon distribution in time. To approximate the integral in Eq. (5), we simply used the rectangular rule, but, of course, more sophisticated approximations may provide more stable solutions.

The number of phonon modes in a volume $V=L^{3}$ is in the order of $10^{11}$ for $\mathrm{L}=1 \mu \mathrm{m}$. This is a too large a number to compute Eq. (6) for each such mode. Fortunately, phonon properties are usually smooth functions of the wavevector $\mathbf{q}$; therefore, we assume that the occupation function can be modeled using $N$ representative phonon modes, $\left(\mathbf{q}_{i}, j_{i}\right), i=1, \ldots, N$. Each such mode is then believed to be representable by a wave packet with an envelop function $\delta$ centered around $\mathbf{r}_{i}$. This gives

$$
n_{\mathbf{q} j}(\mathbf{r}, t)=\sum_{i=1}^{N} n_{i}(t) \delta\left(\mathbf{r}-\mathbf{r}_{i}\right) \delta_{\mathbf{q q}_{i}} \delta_{j j_{i}}
$$

where $n_{i}$ is the number of phonons per mode $i$ and $\delta_{\mathbf{q q}_{i}}$ and $\delta_{j j_{i}}$ are Kronecker delta over the wavevectors and band indices, respectively. Within the linearized Boltzmann equation approximation, the collision integral is a linear functional of the occupation function. ${ }^{1}$ Therefore, it can be represented the same way, i.e.,

$$
\left.\frac{\partial n_{\mathbf{q} j}}{\partial t}\right|_{c o l}(\mathbf{r}, t)=\left.\sum_{i=1}^{N} \frac{\partial n_{i}}{\partial t}\right|_{c o l}(t) \delta\left(\mathbf{r}-\mathbf{r}_{i}\right) \delta_{\mathbf{q q}_{i}} \delta_{j j_{i}} .
$$

Substituting Eqs. (7) and (8) into Eq. (6) and assuming that the envelop functions do not overlap, we obtain

$$
n_{i}\left(t_{0}+\delta t\right)=n_{i}\left(t_{0}\right)+\left[\left.\frac{\partial n_{i}}{\partial t}\right|_{c o l}\left(t_{0}\right)\right] \delta t .
$$

If the single mode relaxation time approximation is used, the above equation becomes

$$
n_{i}\left(t_{0}+\delta t\right)=\left(1-\frac{\delta t}{\tau_{i}}\right) n_{i}\left(t_{0}\right)+\frac{\delta t}{\tau_{i}} n_{i}^{0}\left(t_{0}\right) .
$$

In our method, Eq. (10) is solved at each simulation time step using the lifetime $\tau_{i}$ given by density functional theory calculations, for all computed phonon modes of cubic and hexagonal silicon; the latter values of $\tau_{i}$ are shown in the supplementary material.

Combining Eqs. (9) and (3) provides a numerical solution of the Boltzmann equation. The wavepacket centers are moved as a function of time according to Eq. (3); this is the advection step, while the number of phonons in such wave packet is updated according to Eq. (9). This is the scattering step.

To proceed further with this algorithm, initial and boundary conditions should be specified. The boundary conditions are discussed below. According to Eq. (7), for the initial conditions, we may choose

$$
n_{\mathbf{q} j}(\mathbf{r}, t=0)=\sum_{i=1}^{N} n_{i}(t=0) \delta\left(\mathbf{r}-\mathbf{r}_{i}\right) \delta_{\mathbf{q q}_{i}} \delta_{j j_{i}},
$$

with $N$ being the randomly chosen positions. The positions of the wavepackets are chosen randomly to avoid any unphysical correlations between the trajectories that may result from a specific arrangement of the positions. $n_{i}(t=0)$ is determined assuming that at $t=0$, the wavepackets belong to a region of volume $V_{0}$ which is at thermodynamic equilibrium at temperature $T_{0}$. The occupation function must therefore fulfil

$$
\int_{V_{0}} d^{3} r \sum_{\mathbf{q} j} n_{\mathbf{q} j}(\mathbf{r}, t=0)=V_{0} \int d \omega g(\omega) n^{0}(\omega),
$$

where $g(\omega)=\sum_{j} \int \frac{d^{3} q}{(2 \pi)^{3}} \delta\left(\omega-\omega_{\mathbf{q} j}\right)$ is the phonon density of states per unit volume. If $N_{0}$ is the number of wave packets within $V_{0}$, we have

$$
\sum_{i=1}^{N_{0}} n_{i}(t=0)=V_{0}\left(\frac{1}{\Omega_{0} \mathcal{N}} \sum_{\mathbf{q} j} n^{0}\left(\omega_{\mathbf{q} j}\right)\right),
$$

where the integral over the Brillouin zone is approximated by a summation over $\mathcal{N}$ points. $\Omega_{0}$ is the volume of the crystal primitive cell. If we choose $N_{0}=\mathcal{N}$, then 


$$
n_{i}(t=0)=\frac{V_{0}}{\Omega_{0} \mathcal{N}} n^{0}\left(\omega_{\mathbf{q} j}\right)
$$

fulfils Eq. (13). This is our model for initial conditions.

We now consider a thin film of variable thickness $0.1 \mu \mathrm{m} \leq L_{z} \leq 40 \mu \mathrm{m}$. We would like to compute the crossplane thermal conductivity, in the $z$ direction. As mentioned above, we need to impose boundary conditions to solve the Boltzmann equation. We chose periodic boundary conditions in the $x$ and $y$ directions. This means that the thin film is partitioned into cells of volume $V=L_{x} L_{y} L_{z}$, repeated periodically up to infinity in the $x$ and $y$ directions. The Boltzmann equation is then solved in only one of those cell, and each time a phonon wavepacket leaves that cell in the $x$ or $y$ directions, it immediately re-enters the cell from the opposite face with the same group velocity. This preserves heat flux in the $z$ direction and does not introduce any resistance effect to phonon transport such as those encountered in nanowires. ${ }^{17}$

The left hand side of Eq. (13) depends on the temperature $T_{0}(\mathbf{r})$ through the Bose Einstein occupation function. Therefore, the temperature field $T_{0}(\mathbf{r})$ should be given to start the calculation. In practice, it is done by dividing the volume $V=L_{x} L_{y} L_{z}$ into subcells of volume $V_{0}=L_{x} L_{y} \Delta L_{z}$, where the temperature field is assumed to be constant, and by applying Eq. (13) locally into such subcell.

We have applied this strategy to compute the lattice thermal conductivity of thin films of cubic or hexagonal silicon using $L_{x}=L_{y}=100 \mathrm{~nm}$. The thickness $L_{z}$ is partitioned into subcells of length $\Delta L_{z}=L_{z} / N_{z}$, with $10 \leq N_{z} \leq 40$, and the initial temperature field is shown in Fig. 1. The temperature difference between the two extreme subcells is fixed to $\Delta T=4 \mathrm{~K}$. The evolution of the temperature field as a function of time, as well as the heat flux, is shown in Fig. 1 between 0 and $5 \mathrm{~ns}$. We can observe that the heat flux is uniform through the system after $5 \mathrm{~ns}$, which means that the system has reached a stationary regime. In the first and last subcells, the flux is zero because these subcells are used to impose the boundary conditions $T_{z=0}=302 \mathrm{~K}$ and $T_{z=L_{z}}=298 \mathrm{~K}$. Therefore, it can be seen that the temperature field does not converge to the linear variation predicted by the heat equation over the whole system but only where the heat flux has reached a stationary value. This shows that to extract the thermal conductivity from the temperature field and heat flux, $\kappa=|J| /|\nabla T|$, we should not use the imposed temperature gradient $|\nabla T| \neq\left(T_{z=L_{z}}-T_{z=0}\right) / L_{z}$ but rather

$$
|\nabla T|=\frac{T_{z=L_{z}-\Delta L_{z}}-T_{z=\Delta L_{z}}}{L_{z}-2 \Delta L_{z}} .
$$

The latter issue becomes important as the length $L_{z}$ gets smaller. In such cases, ballistic transport through the thin film increases, and the imposed temperature gradient is drastically reduced. For instance, for $L_{z}=100 \mathrm{~nm},|\nabla T|=23 \times 10^{6} \mathrm{~K} / \mathrm{m}$, instead of an imposed value of $40 \times 10^{6} \mathrm{~K} / \mathrm{m}$. The thermal conductivities we obtain are plotted in blue in Fig. 2 as a function of $L_{z}$, for cubic and hexagonal silicon. For hexagonal silicon, two cases are considered, as indicated with the $[a]$ and $[c]$ symbols. $[a]$ means that the a lattice vector of the primitive unit cell of hexagonal silicon has been chosen to be along the $z$ direction, while
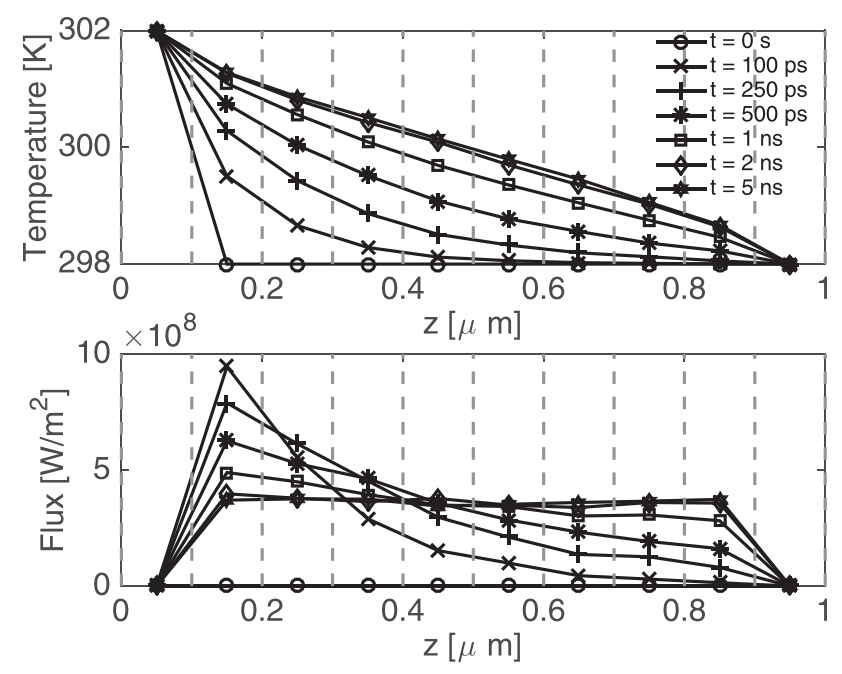

FIG. 1. Temperature profile and energy flux at different times for cubic silicon at $300 \mathrm{~K}$ with $L_{z}=1 \mu \mathrm{m}$. The time step was chosen to be $\delta t=0.1 \mathrm{ps}$. Vertical dashed lines are used to represent the subcells.

$[c]$ means that the $\mathbf{c}$ lattice vector was used. This way, the crystalline anisotropy can be considered.

Moreover, we have also computed the thermal conductivity for a few thin films with the particle Monte Carlo method of Refs. 11-14 but using our ab initio data as inputs. The results of these computations are shown as dashed grey lines in Fig. 2. The particle Monte Carlo method, within our implementation, slightly overestimates the bulk value of cubic and hexagonal silicon, while the method we propose, based on characteristic lines, gives good agreement. Indeed, in the method we propose, the effects of collisions on the phonon occupation function are rigorously considered through Eq. (9), and no special care is needed to fulfil conservation of energy or momentum.

Assuming a Debye spectrum and $\tau^{a} \propto \omega^{-2},{ }^{19}$ an analytical estimate for the thermal conductivity of films can be obtained. ${ }^{18}$ This corresponds to the full grey curves shown in

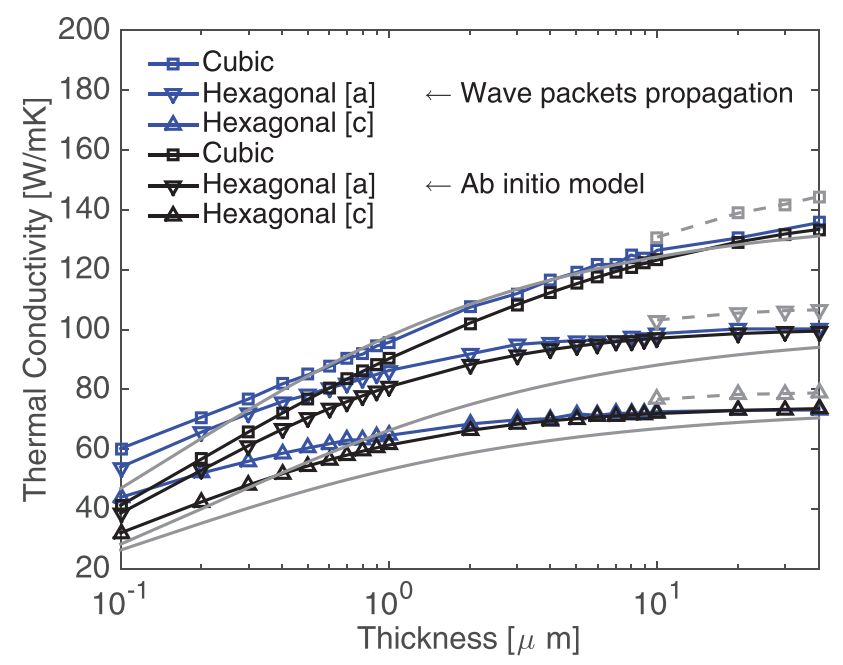

FIG. 2. Lattice thermal conductivity, as a function of thickness, for cubic and hexagonal silicon. The results of the numerical method of II are shown in blue, while the black curves correspond to the ab initio model, Eq. (14). The dashed grey curves correspond to a Monte Carlo solution of Boltzmann equation using ab initio data as input, as detailed in Refs. 11-14. The full grey curve corresponds to the thermal conductivity obtained in the Debye approximation. $^{18}$ 


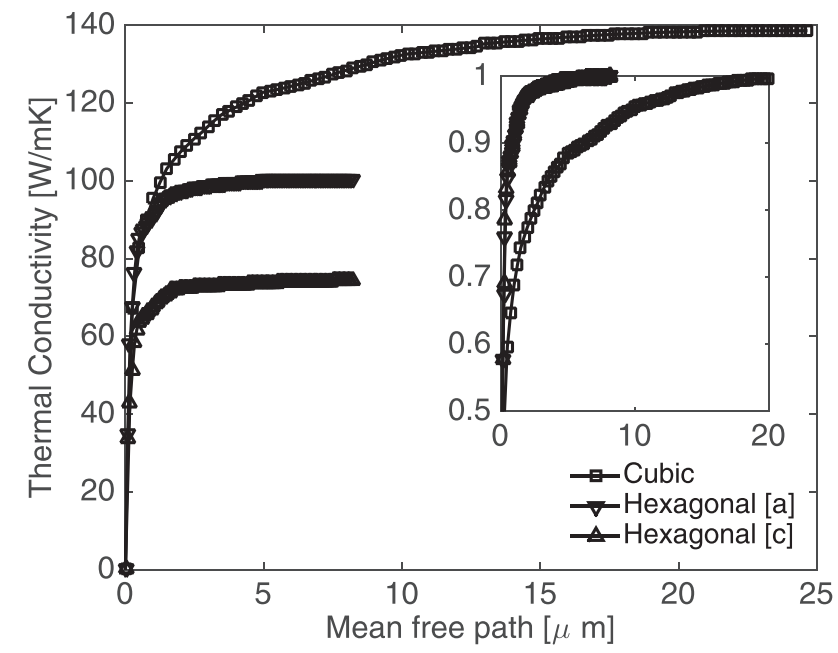

FIG. 3. Cumulative $a b$ initio lattice thermal conductivity, as a function of the phonon mean free path. The inset shows the cumulative thermal conductivity, normalized to 1 . The curves for hexagonal silicon along the $a$ and $c$ axes are almost identical.

Fig. 2, using the sound velocity in the $z$ direction of 4232, 4349 , and $4971 \mathrm{~m} / \mathrm{s}$ for cubic silicon and hexagonal silicon in the $[a]$ and $[c]$ orientations, respectively. Clearly, this simple model is a reasonable approximation, but it cannot be considered to be a quantitative one when anisotropy needs to be considered. Moreover, it should be noted that to get those results, we have used the average value of the $z$ component of the velocity vectors of longitudinal and transverse modes and not the average value of the modulus of the velocity vectors, as it is usually done for the Debye model.

For the three cases we consider, we observe that the thermal conductivity decreases as the thin film thickness is decreased. This is well understood and can be attributed to the reduction of the phonon mean free path because of the scattering on the boundaries. We can also observe that the thermal conductivity of cubic silicon decreases much more rapidly than the one of hexagonal silicon, in either the $a$ or $c$ orientation. Indeed, in a $2 \mu \mathrm{m}$ thick film, the bulk value of cubic silicon has been reduced by $22 \%$, while the reduction is only of $8 \%$ in hexagonal silicon. The cumulative lattice thermal conductivity, plotted as a function of the phonon mean free path $\left|\boldsymbol{\Lambda}_{\mathbf{q} j}\right|$ $=\tau_{\mathbf{q} j}\left|\mathbf{v}_{\mathbf{q} j}\right|$ and computed using ab initio data, is presented in Fig. 3. The phonons with the mean free path less than $2 \mu \mathrm{m}$ contribute $77 \%$ of the total thermal conductivity in cubic silicon and $97 \%$ of the total thermal conductivity in hexagonal silicon. This explains the results of Fig. 2. Indeed, this means that in hexagonal silicon, most of the phonons contributing to the thermal conductivity have a mean free path much shorter than $2 \mu \mathrm{m}$, due to interactions with other phonons. This makes scattering on boundaries only a marginal event. On the contrary, for phonons with a longer mean free path, scattering on boundaries is one of the few interaction events experienced by phonons and therefore contributes markedly, leading to a reduction of thermal conductivity. This argument can be made more quantitative. Indeed, if we consider the collision time with the boundary to be position-independent and if $\theta$ is the angle of $\mathbf{v}_{\mathbf{q} j}$ with the $z$ axis, then we can write $\tau_{\mathbf{q} j}^{b}\left|\mathbf{v}_{\mathbf{q} j}\right|=L_{z} /(2 \cos \theta)$, where $\tau_{\mathbf{q} j}^{b}$ is the phonon scattering time on the boundaries. This gives $1 / \tau_{\mathbf{q} j}^{b}=2\left|\mathbf{v}_{\mathbf{q} j}^{z}\right| / L_{z}$. Assuming the interactions with the phonons and boundaries to be independent, we obtain for the total lifetime, $1 / \tau_{\mathbf{q} j}=1 / \tau_{\mathbf{q} j}^{a}+1 / \tau_{\mathbf{q} j}^{b}$, which can be used with the well know equation for the thermal conductivity of a bulk material $^{2}$

$$
\kappa_{0}=\frac{1}{\Omega_{0} \mathcal{N}} \sum_{\mathbf{q} j} \tau_{\mathbf{q} j} \mathbf{v}_{\mathbf{q} j}^{z} \mathbf{v}_{\mathbf{q} j}^{z}\left(\hbar \omega_{\mathbf{q} j} \frac{\partial n_{\mathbf{q} j}^{0}}{\partial T}\right) .
$$

The results of these calculations are plotted in black curves in Fig. 2. Clearly, this very simple model is in reasonable agreement with the numerical solution of the Boltzmann equation in the simple geometry we consider. The agreement is almost perfect at a large thickness, where the scattering on boundary is less important, but becomes more approximate below $2 \mu \mathrm{m}$. In this range, as expected, the details of the scattering become important.

It should be noted that as long as the thin thickness remains of the order of the average phonon mean free path in the $z$ direction $[\approx 155 \mathrm{~nm}$ for cubic silicon and 115 and $94 \mathrm{~nm}$ for hexagonal silicon in the $[a]$ and $[c]$ orientations, at $300 \mathrm{~K}$ (Ref. 22)], the value of the lattice thermal conductivity is limited by the scattering of the phonons on the boundaries, the so called Casimir limit. This is the case we have considered in this paper. Below such a limit, when the system size becomes comparable to the characteristic wavelength of phonons, phonon confinement effects should occur. This leads to strong modifications of the phonon dispersion relations and lifetimes, ${ }^{20}$ which are not captured in our model, since we are using the bulk phonon properties. The dominant wavelength of the phonons can be estimated using $\lambda_{d} \sim h c / k_{B} T .^{21}$ Taking $9000 \mathrm{~m} / \mathrm{s}$ as an upper limit to the sound velocity $c$, we obtain $\lambda_{d}=43 \mathrm{~nm}$ at $10 \mathrm{~K}$ and $\lambda_{d}=1.4 \mathrm{~nm}$ at room temperature. $L_{z}$ should be greater than $\lambda_{d}$ for the results of Fig. 2 to be considered as accurate. Obviously, at such a small length scale, the validity of the semiclassical Boltzmann equation we are using should also be questioned.

To summarize, in this work, we have proposed a method to solve the phonon Boltzmann transport equation parametrized with $a b$ initio calculations. The method has then been implemented and is applied to compute the thermal conductivity of cubic and hexagonal silicon thin films. In particular, it allowed us to bring out the rapid decrease in the thermal conductivity with the thin film thickness and to evidence the differences between cubic and hexagonal silicon. In cubic silicon, the mean free path of heat carrying phonons is longer than that of hexagonal silicon; therefore, the thermal conductivity drops more rapidly as the thickness is reduced. This has been obtained quantitatively by solving the Boltzmann equation, evidencing the applicability of the method. Moreover, the method we propose can be applied to any device geometries and materials. Therefore, we believe it to be useful for a better understanding of heat transport at the micron scale. This is the purpose of future works.

See supplementary material for the details of the $a b$ initio calculations.

This work was performed using HPC resources from GENCI-IDRIS (Grant No. 2015-x2014097186). 
${ }^{1}$ L. Chaput, Phys. Rev. Lett. 110, 265506 (2013).

${ }^{2}$ A. Togo, L. Chaput, and I. Tanaka, Phys. Rev. B 91, 094306 (2015).

${ }^{3}$ G. Fugallo, M. Lazzeri, L. Paulatto, and F. Mauri, Phys. Rev. B 88, 045430 (2013).

${ }^{4}$ S. Narasimhan and D. Vanderbilt, Phys. Rev. B 43, 4541 (1991).

${ }^{5}$ L. Chaput, A. Togo, I. Tanaka, and G. Hug, Phys. Rev. B 84, 094302 (2011).

${ }^{6}$ K. Esfarjani, G. Chen, and H. T. Stokes, Phys. Rev. B 84, 085204 (2011).

${ }^{7}$ A. Debernardi, S. Baroni, and E. Molinari, Phys. Rev. Lett. 75, 1819 (1995).

${ }^{8}$ A. Seko, A. Togo, H. Hayashi, K. Tsuda, L. Chaput, and I. Tanaka, Phys. Rev. Lett. 115, 205901 (2015).

${ }^{9}$ G. E. W. Bauer, E. Saitoh, and B. J. van Wees, Nat. Mater. 11, 391 (2012).

${ }^{10}$ D. Rowe, Thermoelectrics Handbook: Macro to Nano (CRC Press, 2005), ISBN: 9781420038903.

${ }^{11}$ R. B. Peterson, J. Heat Transfer 116, 815 (1994).

${ }^{12}$ S. Mazumder and A. Majumdar, J. Heat Transfer 123, 749 (2001).
${ }^{13}$ D. Lacroix, K. Joulain, and D. Lemonnier, Phys. Rev. B 72, 064305 (2005).

${ }^{14}$ Q. Hao, G. Chen, and M.-S. Jeng, J. Appl. Phys. 106, 114321 (2009).

${ }^{15}$ B. Vermeersch, J. Carrete Montaña, and N. Mingo, Appl. Phys. Lett. 108, 193104 (2016).

${ }^{16}$ I. Sneddon, Elements of Partial Differential Equations, Dover Books on Mathematics Series (Dover Publications, 2006), ISBN: 9780486452975.

${ }^{17}$ D. Lacroix, K. Joulain, D. Terris, and D. Lemonnier, Appl. Phys. Lett. 89, 103104 (2006).

${ }^{18}$ A. J. H. McGaughey, E. S. Landry, D. P. Sellan, and C. H. Amon, Appl. Phys. Lett. 99, 131904 (2011).

${ }^{19}$ P. G. Klemens, Solid State Physics (Academic Press, 1958), Vol. 7.

${ }^{20}$ A. Balandin and K. L. Wang, Phys. Rev. B 58, 1544 (1998).

${ }^{21}$ R. Chen, A. I. Hochbaum, P. Murphy, J. Moore, P. Yang, and A. Majumdar, Phys. Rev. Lett. 101, 105501 (2008).

${ }^{22}$ It is different from the average of the modulus of the phonon mean free path vectors, which, for example, is about $300 \mathrm{~nm}$ in cubic silicon. 